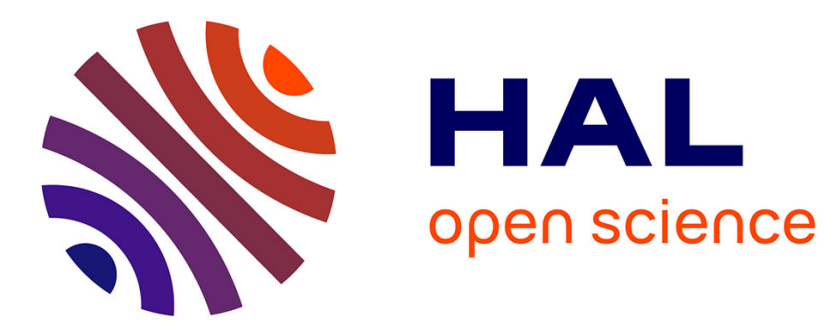

\title{
Inverse Scattering Using a Time Reversal RADAR
}

Lucio Bellomo, K. Belkebir, Marc Saillard, Sebastien Pioch, P. C. Chaumet

\section{To cite this version:}

Lucio Bellomo, K. Belkebir, Marc Saillard, Sebastien Pioch, P. C. Chaumet. Inverse Scattering Using a Time Reversal RADAR. EMTS, Aug 2011, Berlin, Germany. hal-00703387

\section{HAL Id: hal-00703387 \\ https://hal.science/hal-00703387}

Submitted on 1 Jun 2012

HAL is a multi-disciplinary open access archive for the deposit and dissemination of scientific research documents, whether they are published or not. The documents may come from teaching and research institutions in France or abroad, or from public or private research centers.
L'archive ouverte pluridisciplinaire HAL, est destinée au dépôt et à la diffusion de documents scientifiques de niveau recherche, publiés ou non, émanant des établissements d'enseignement et de recherche français ou étrangers, des laboratoires publics ou privés. 


\title{
Inverse Scattering Using a Time Reversal RADAR
}

\author{
Lucio Bellomo ${ }^{\# 1}$, Kamal Belkebir ${ }^{* 2}$, Marc Saillard ${ }^{* 3}$, Sébastien Pioch ${ }^{\# 4}$, Patrick Chaumet ${ }^{* 5}$ \\ \# LSEET, UMR CNRS 6017 \\ Université du Sud Toulon-Var, Bâtiment F, BP 20132, 83957 La Garde Cedex, France \\ ${ }^{1-3}$ \{lucio.bellomo, marc.saillard, sebastien.pioch\}@lseet.univ-tln.fr \\ * Institut Fresnel, UMR CNRS 6133 \\ Campus de Saint Jérôme, case 162, 13397 Marseille Cedex 20, France \\ ${ }^{4-5}$ \{kamal.belkebir,patrick.chaumet\}@fresnel.fr
}

\begin{abstract}
This work presents a new RADAR prototype built for the purpose of imaging targets located in a cluttered environment. The system is capable of performing Phase Conjugation experiments in the ultrawideband [2-4] GHz. In addition, applying the D.O.R.T. method to the inter-element matrix allows us to selectively focus onto targets, hence reducing the clutter contribution. We aim to experimentally explore the use of this focusing wave into an inversion algorithm, in order to improve its robustness against noise.
\end{abstract}

\section{INTRODUCTION}

Using electromagnetic waves for the characterization of otherwise inacessible objects is of interest in many applicative contexts where non-invasive and non-destructive investigations are required, such as for instance medical imaging, geophysical and geological probing, etc. With this purpose, robust nonlinear inversion algorithms minimizing the difference between the measured scattered field and the one relative to the reconstructed objects have been built. In [1] experimental time-harmonic data have been succesfully inversed through a number of such schemes.

Nevertheless the inverse scattering problem is known to be ill-posed and not to have a unique solution. Therefore any clutter present in the investigation region might have a strong impact on the final result. One way to reduce this effect consists in using within the inversion algorithm the response of the scatterer to an incident field focusing onto it. The Time Reversal [2] technique and, more specifically, the D.O.R.T. method [3] have in the last decade proven to be very effective for this purpose [4].

The D.O.R.T. method has succesfully been used as a regularization term in an inversion scheme [5], as applied to synthetic data. In order to experimentally prove such results and further explore the potential of the approach, a Time Reversal RADAR is presently under construction. The system consists in a linear array of 8 antennas plus one more spare antenna working in the ultrawideband [2-4] GHz. Besides recording the $8 \times 8$ inter-element matrix, the prototype can physically re-emit the focusing wave issued from the D.O.R.T. method, so that the medium response to it can also be measured and eventually included in the inversion process.
This paper describes briefly the inversion scheme including the D.O.R.T. regularization and presents some preliminary experimental results validating the RADAR. More advanced results will be presented at the conference.

\section{INVERSION PROCEDURE INCLUDING D.O.R.T. TERM}

The two-dimensional inverse scattering problem is stated in the frequency domain, where for each frequency $\omega_{p}, p=$ $1, \ldots, P$ and for each illuminating source $j=1, \ldots, J$, the scattering problem may be formulated as two coupled contrast-source integral equations involving the total electric field $E_{j, p}$ and the contrast distribution $\chi_{p}(\mathbf{r})=\varepsilon_{r ; p}(\mathbf{r})-1$, with $\varepsilon_{r ; p}$ being the complex relative permittivity. For the sake of simplicity, symbolic operator notations are used:

$$
\begin{gathered}
E_{j, p}^{\mathrm{d}}=\mathbf{G}_{j, p}^{\Gamma} \chi_{p} E_{j, p}, \\
E_{j, p}=E^{\mathrm{inc}}+\mathbf{G}_{j, p}^{\Omega} \chi_{p} E_{j, p},
\end{gathered}
$$

where $E, E^{\mathrm{inc}}$, and $E^{\mathrm{d}}$ denote the total, incident, and scattered fieds, respectively. $\mathbf{G}^{U=\Gamma, \Omega}$ represents an integral operator whose kernel involves the two-dimensional free space Green function. The aim is to determine the permittivity distribution in a bounded box $\Omega$, such that the corresponding scattered field matches the one measured along a measurement line $\Gamma$, $E^{\text {d;meas }}$. An iterative approach is used to solve this ill-posed and nonlinear problem [6]. In this approach, starting from an initial guess, the parameter of interest, i.e. the permittivity distribution, is gradually adjusted by minimization of a cost function $\mathcal{F}$ of the form

$$
\begin{aligned}
\mathcal{F}\left(E_{j, p} ; \chi_{p}\right) & =\frac{\sum_{j=1}^{J} \sum_{p=1}^{P}\left\|h_{j, p}^{(1)}\right\|_{\Omega}^{2}}{\sum_{j=1}^{J} \sum_{p=1}^{P}\left\|E_{j, p}^{\mathrm{inc}}\right\|_{\Omega}^{2}} \\
& +\frac{\sum_{j=1}^{J} \sum_{p=1}^{P}\left\|h_{j, p}^{(2)}\right\|_{\Gamma}^{2}}{\sum_{j=1}^{J} \sum_{p=1}^{P}\left\|E_{j, p}^{\mathrm{d} ; \text { meas }}\right\|_{\Gamma}^{2}}
\end{aligned}
$$

where the residual errors $h^{(1)}$ and $h^{(2)}$ are defined as follows:

$$
\begin{gathered}
h^{(1)}=E_{j, p}^{\mathrm{inc}} E_{j, p}+\mathbf{G}_{j, p}^{\Omega} \chi_{p} E_{j, p}, \\
h^{(2)}=E_{j, p}^{\mathrm{d} ; \text { meas }} \mathbf{G}_{j, p}^{\Gamma}, \chi_{p} E_{j, p} .
\end{gathered}
$$


Thanks to the D.O.R.T. method [3] one can retrieve the amplitude and phase laws needed by the array to generate a wave focusing onto a target. Such a wave can therefore be considered as an additional incident wave, $E^{\text {inc;DORT }}$, to which is associated the corresponding diffracted field $E^{\mathrm{d} ; \mathrm{DORT}}$. The idea is then to construct a new cost function $\mathcal{F}^{\text {DORT }}\left(E_{p}^{\text {DORT }} ; \chi_{p}\right)$ built exactly as in $(3)$ but based on these D.O.R.T. fields, and to merge it with the "regular" one as a regularization term:

$$
\begin{aligned}
\tilde{\mathcal{F}}\left(E_{j, p}, E_{p}^{\mathrm{DORT}} ; \chi_{p}\right)= \\
\mathcal{F}\left(E_{j, p} ; \chi_{p}\right) \cdot \mathcal{F}^{\mathrm{DORT}}\left(E_{p}^{\mathrm{DORT}} ; \chi_{p}\right) .
\end{aligned}
$$

To improve the efficiency of the algorithm, we add $a$ priori information stating that the desired electrical susceptibility must be greater than unity and the conductivity to be positive. With these conditions and assuming an Ohmic dispersion model for materials of interest, the contrast function $\chi_{p}$ reads as

$$
\chi_{p}=\xi^{2}+i \frac{\eta^{2}}{\omega_{p} \varepsilon_{0}} .
$$

The minimization of the cost function (6) with respect to $\xi$ and $\eta$ is accomplished using a modified gradient-like method [6].

\section{RADAR DESCRIPTION AND PRELIMINARY RESULTS}

The architecture of our RADAR (Fig. 1) is built around a 2-port Vector Network Analyzer serving both as signal source and receiver. The RF front-end is made of a linear array of $8 \mathrm{UWB}$ antennas $\left(\mathrm{A}_{1}-\mathrm{A}_{8}\right)$ plus one more spare antenna $\left(\mathrm{A}_{9}\right)$. They are antipodal symmetric Exponentially Tapered Slot Antennas (ETSA) radiating a vertically-polarized (direction perpendicular to the plane of Fig. 1) electric field. To be able to experimentally re-transmit a focusing wave, each array channel is controlled both in amplitude and phase via wideband attenuator/phase shifter $(\mathrm{A} / \Phi)$ couples driven numerically. Antennas and phase shifters respectively impose the low and high boundaries of the exploitable [2-4] $\mathrm{GHz}$ frequency band, sampled with a step of $10 \mathrm{MHz}$. The spacing between the array antennas has been set to $5 \mathrm{~cm}\left(\lambda_{3} \mathrm{GHz} / 2\right)$ for a best compromise between antenna coupling and spatial sampling of the scattered field. Also, differential measurements (difference between measurements with and without the scatterer in place) are always used in order to further reduce antenna coupling [7]

\section{A. Generation of a focusing wave}

One of the experiments we have conducted to validate the prototype consists in applying the D.O.R.T. method in a configuration with a $4 \mathrm{~cm}$-diameter metallic cylinder located $40 \mathrm{~cm}$ away from the array center. The array antennas transmit and receive at turn, to finally build the $8 \times 8$ so-called interelement matrix. In Fig. 2(a), we have plotted the three largest eigenvalues versus frequency; the largest one is effectively associated to the target, and the corresponding eigenvector supplies the amplitude and phase laws needed to generate a

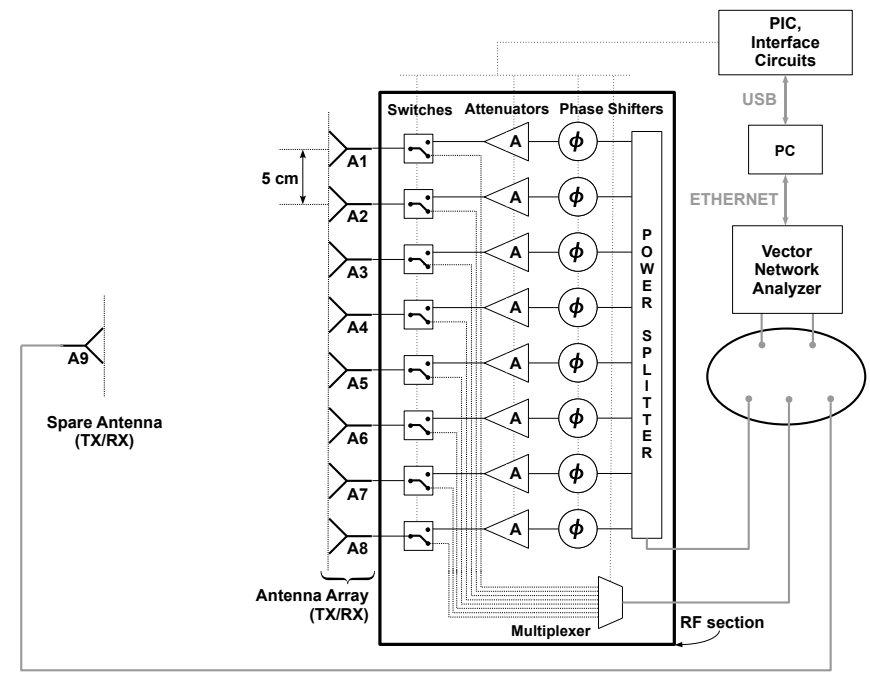

Fig. 1. Prototype architecture.

wave focusing onto it. We have experimentally coded such vector at each frequency into the system and we have measured the object response to the focusing wave. Switching to the time domain through a Fourier transform, we have also simulated the propagation of the focusing wave and built a chart of the field over the area of interest. Here, the antennas are simply modeled as vertical electric dipoles. The frame at the instant when the wave converges onto the cylinder is given in Fig. 2(b), where an excellent focusing can be appreciated.

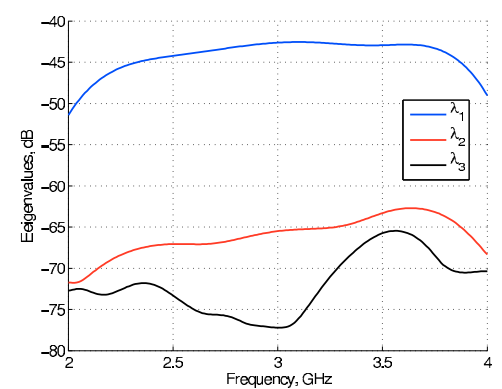

(a)

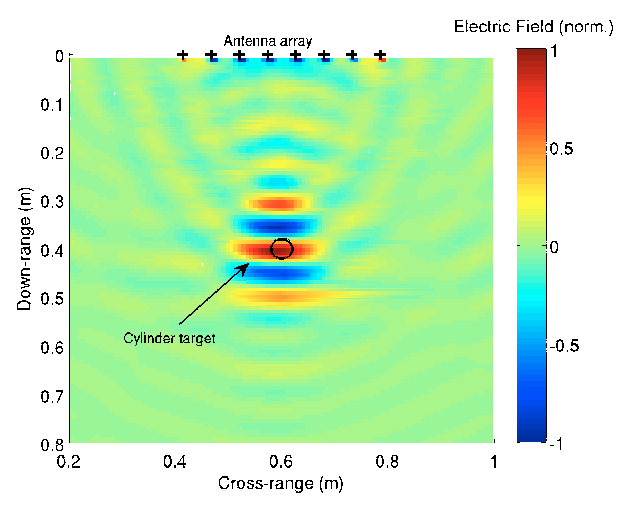

(b)

Fig. 2. UWB D.O.R.T. experiment. (a) Array inter-element matrix eigenvalues distribution versus frequency and (b) one frame of the synthetic timedomain field chart movie associated to the largest eigenvalue. 


\section{B. Inverse Scattering}

We have started testing the RADAR in the framework of $2 \mathrm{D}$ inverse scattering. The configuration is depicted in Fig. 3. The target is the same metallic cylinder as above, located in front of antenna $A_{5}$. Notice the small array aperture angle, $\sim 34^{\circ}$.

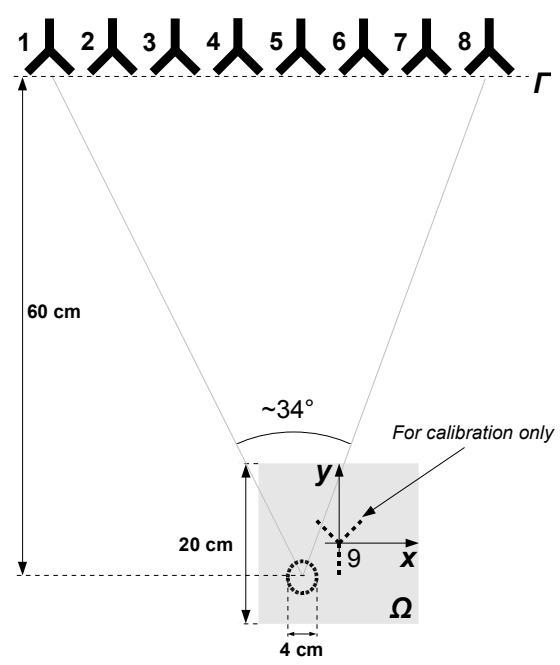

Fig. 3. Experimental configuration of the inverse scattering problem.

1) Calibration of the Incident Field: First of all, an accurate calibration of the incident field is required. Our setup is such that the outer antennas $\left(\mathrm{A}_{1}\right.$ and $\left.\mathrm{A}_{8}\right)$ do not illuminate the target in the forward direction, but with incidence angle up to $\sim 20^{\circ}$. This, in conjunction with the anisotropic antenna radiation patterns, make an accurate incident field modeling compulsory.

For calibration purpose, we place the spare antenna A9 in front of the array at the center of the test region $\Omega$ (Fig. 3). The radiated field measured by each antenna $\mathrm{A}_{j}$ of the array is stored in the vector of transmission S-parameters $\mathbf{S}^{\text {cal }}$, whose $j^{\text {th }}$ component $S_{j 9}^{\mathrm{cal}}, j=1, \ldots, J=8$ is recorded at each frequency $\omega_{p}, p=1, \ldots, P=201$ in the [2-4] $\mathrm{GHz}$ band (we drop from here on the subscript $p$ for lighter notations). Then, as in [8], we use a Fourier expansion to model the radiation pattern:

$$
S_{j 9}^{\mathrm{cal}}=\sum_{n=-N}^{N} \gamma_{n} H_{n}^{-}\left(k r_{j 9}\right) e^{-i n \theta_{j 9}}, \quad j=1, \ldots, 8
$$

or, with a matrix notation,

$$
\mathbf{S}^{\mathrm{cal}}=\mathbf{H} \boldsymbol{\Gamma},
$$

where $k$ is the wave number in free-space at the $p^{\text {th }}$ frequency, $\gamma_{n}$ is the $n^{\text {th }}$ unknown coefficient, $H_{n}^{-}$is the Hankel function of second kind of order $n$ and $r_{j 9}$ and $\theta_{j 9}$ are the polar coordinates of the vector going from $\mathrm{A}_{9}$ to $\mathrm{A}_{j}$ (Fig. 3).

The choice of the truncation order $N$ is delicate: if too high, the highest coefficients of $\mathbf{S}^{\text {cal }}$ are corrupted by noise and can affect the solution of the inversion algorithm, whereas if it is too low, it fails at modeling the pattern away from the forward direction. Plotting the $\gamma_{n}$, it appears that they decrease rapidly for $N>1$, so that $N=1$ is a choice well suited for our experimental setup. Eq. (9) can finally be solved for $\Gamma$ by computing the pseudo-inverse of $\mathbf{H}$ through its SVD [8].

One must notice though that in (9) $\boldsymbol{\Gamma}$ is experimentally related to the radiation pattern of both A9 and the array antennas, that is, the transmitting and receiving antennas. Nevertheless, in [6], [8] it is used to model the incident field only. We propose to split it in two by introducing a new quantity, the effective length of an antenna $l_{\mathrm{e}}(r, \theta)$, which we lend from classical Antenna Theory [9] where it is used to describe the far-field radiation pattern of an antenna. Under the assumption that the antennas are all identical, for each point in $\Omega$ and for each antenna we can express the antenna radiation pattern as a function of the square of $l_{\mathrm{e}}$ :

$$
\sum_{n=-N}^{N} \gamma_{n} H_{n}^{-}\left(k r_{j}\right) e^{-i n \theta_{j}}=l_{\mathrm{e}}^{2}\left(r_{j}, \theta_{j}\right) H_{0}^{-}\left(k r_{j}\right),
$$

where $r_{j}$ and $\theta_{j}$ are the polar coordinates of the vector going from $\mathrm{A}_{j}$ to the chosen point.

Once all the $l_{e}\left(r_{j}, \theta_{j}\right)$ have been determined from (10) the Green function $G^{\Gamma}$ and the incident field from the transmitting antenna $\mathrm{A}_{j}$ in (1) and (2) are approximated by

$$
E_{j}^{\mathrm{inc}}=l_{\mathrm{e}}\left(r_{j}, \theta_{j}\right) H_{0}^{-}\left(k r_{j}\right)
$$

and

$$
\tilde{G}_{j}^{\Gamma}=l_{\mathrm{e}}\left(r_{j}, \theta_{j}\right) G_{j}^{\Gamma}
$$

respectively, thus creating two separate transmission and reception antenna radiation patterns. Except from these "initial" modifications, the inversion algorithm remains unchanged.

In order to appreciate the impact of this calibration strategy, we have simulated the scattered field associated to the problem depicted in Fig. 3 with the domain integral formalism. Using $\mathrm{A}_{5}$ as transmitting antenna, Fig. 4 shows the comparison between the measured and the simulated scattered fields as a function of the frequency (vertical axis) and of the number of the receiving antenna (horizontal axis). The effect of the calibration is quite obvious when amplitude is concerned: measurements are reported in Fig. 4(a), whereas the next three plots depict the simulated results for three different calibration methods: (b) a monopolar ( $N=0$ in (8)) and (c) a multipolar $(N=1)$ expansion applied to the incident field model without splitting $\Gamma$ in transmission and reception effective lengths, and (d) the same multipolar expansion but including the concept of effective length as in (10). It is noticed that the last technique outperforms the two others because it is the only technique that appropriately models the reception gain of the outer antennas, which indeed "see" the target away from the forward direction. The phase modeling is less critical and all methods give an excellent phase matching between measurements and simulations. Here, we only report the result when the effective lengths are used (Fig. 4(f)). 


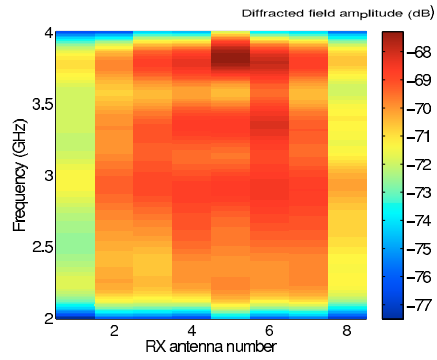

(a)

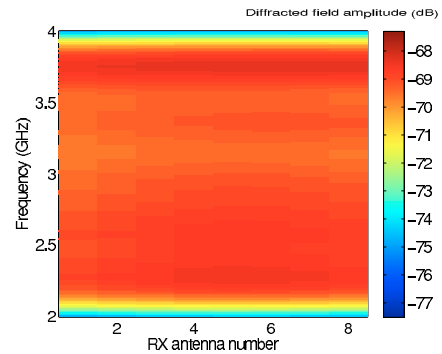

(c)

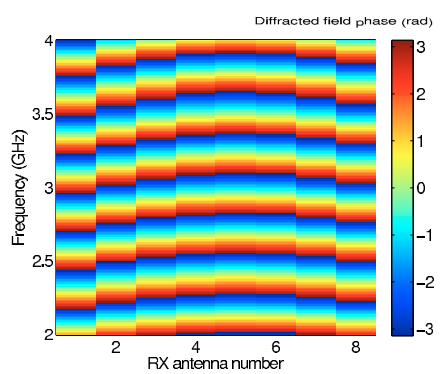

(e)

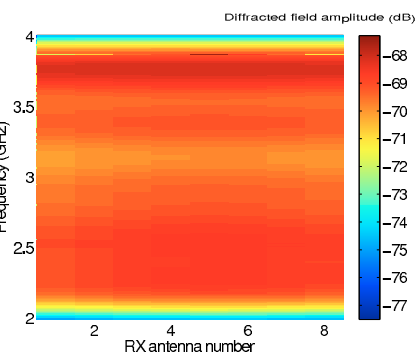

(b)

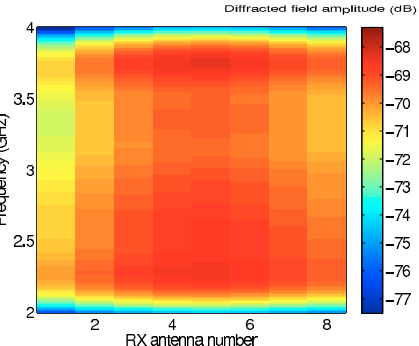

(d)

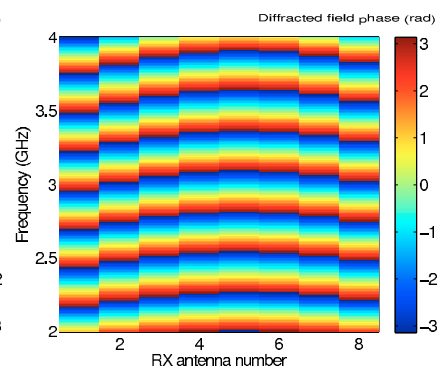

(f)
Fig. 4. (a-d) Amplitude and (e-f) phase of the diffracted field as a function of the frequency and of the number of the receiving antenna in the case of antenna $A_{5}$ used as emitter. (a,e) Measurement; simulation with incident field calibration based on a (b) monopolar, (c) multipolar $(N=1)$ and (d,f) multipolar/effective length expansion.

2) Reconstruction Results: The inverse problem is based on the algorithm previously described but does not include the response to the D.O.R.T. focusing wave yet. We look for the real and imaginary permittivity profiles in the $20 \mathrm{~cm} \times 20 \mathrm{~cm}$ test region $\Omega$, discretized with a step of $\sim 0.65 \mathrm{~cm}\left(\sim \lambda_{3} \mathrm{GHz} / 15\right)$, and no assumption is made about the material (dielectric, conductor) of the target. The initial guess is derived from the backpropagation method [10]. In Fig. 5 are shown the conductivity distributions provided by the inversion scheme when carried out with synthetic data on one hand (left), and with experimental data on the other hand (right). In the latter case, the effective length calibration technique is applied. The real part of the permittivity is not shown since it is noisy and small-valued everywhere in $\Omega$. Also, the array aperture is so small that we cannot image the back of the object, as confirmed by the inversion from noiseless synthetic data, which match that from measurements very well, except that the maximum of conductivity reaches $0.9 \mathrm{~S} / \mathrm{m}$ instead of $0.4 \mathrm{~S} / \mathrm{m}$.

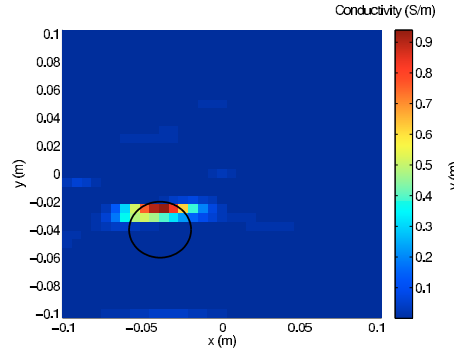

(a)

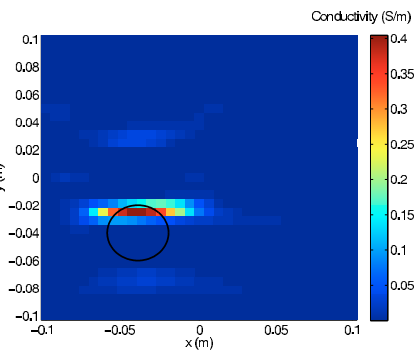

(b)
Fig. 5. Distribution of the reconstructed conductivity from (a) synthetic data (b) experimental data.

\section{Conclusion And Perspectives}

We have manufactured a Time Reversal RADAR prototype working in the [2-4] $\mathrm{GHz}$ frequency band. Its validation has been initially accomplished in the context of the D.O.R.T. method used for target detection and localization. In the framework of 2D inverse scattering, we have succesfully imaged a metallic cylinder despite the finite height of the target and the unmodeled anistropic radiation pattern of the antennas along the vertical axis. We are currently building a second antenna array that will allow us to measure the response to the focusing wave issued from the D.O.R.T. processing. Such a response will be used to improve the inversion results in cluttered media.

\section{ACKNOWLEDGMENT}

This work is supported by DGA/CNRS. The ETSA antennas have been kindly offered by LEAT in Nice-Sophia Antipolis, France.

\section{REFERENCES}

[1] K. Belkebir and M. Saillard, "Special section on testing inversion algorithms against experimental data: inhomogeneous targets," Inverse Problems, vol. 21, no. 6, pp. S1-S3, Dec. 2005.

[2] M. Fink, C. Prada, F. Wu, and D. Cassereau, "Self focusing in inhomogeneous media with time reversal acoustic mirrors," in Proc. IEEE Ultrasonics Symposium, vol. 2, Montreal, Que., Canada, Oct. 1989, pp. 681-686.

[3] C. Prada and M. Fink, "Eigenmodes of the time reversal operator: A solution to selective focusing in multiple-target media," Wave Motion, vol. 20, pp. 151-163, Sep. 1994.

[4] G. Micolau and M. Saillard, "D.O.R.T. method as applied to electromagnetic subsurface sensing," Radio Sci., vol. 38, no. 3, pp. S63-S79, May 2003.

[5] A. Dubois, K. Belkebir, and M. Saillard, "Localization and characterization of two-dimensional targets buried in a cluttered environment," Inv. Probl., vol. 20, no. 6, pp. S63-S79, Nov. 2004.

[6] _ "Retrieval of inhomogeneous targets from experimental frequency diversity data," Inverse Problems, vol. 21, no. 6, pp. S65-S79, Dec. 2005.

[7] A. Cresp, I. Aliferis, M. J. Yedlin, J.-Y. Dauvignac, and C. Pichot, "Time-domain processing of electromagnetic data for multiple-target detection," AIP Conf. Proc., 3rd Conference on Mathematical Modeling of Wave Phenomena, vol. 1106, no. 1, pp. 204-213, Mar. 2009.

[8] D. Franceschini, M. Donelli, and A. Massa, "On the effects of the electromagnetic source modeling in the iterative multiscaling method," Radio Sci., vol. 42, p. RS3020, Jun. 2007.

[9] D. M. Pozar, Microwave engineering, 2nd ed., 1998, pp. 206-208.

[10] R. E. Kleinman and P. M. van den Berg, "Two-dimensation location and shape reconstruction," Radio Sci., vol. 29, no. 4, pp. 1157-1169, 1994. 